\title{
Adjuvant treatment of severe acute pancreatitis with C1 esterase inhibitor concentrate after haematopoietic stem cell transplantation
}

\author{
D T Schneider, W Nürnberger, H Stannigel, H Bönig, U Göbel
}

\begin{abstract}
Background-With an incidence of $4 \%$, acute pancreatitis is a common complication of bone marrow or peripheral haematopoietic stem cell transplantation, which contributes significantly to morbidity and mortality in these patients. In most cases, the pathogenesis of acute pancreatitis cannot be attributed to a single pathogenetic factor, as treatment toxicity, acute graft versus host disease, infection, and cholestasis may all contribute. Acute pancreatitis is characterised by inflammation and activation of digestive proenzymes leading to autodigestive destruction of the pancreas and systemic activation of protease cascades including the complement system.
\end{abstract}

Aim-To describe the effects of human C1 esterase inhibitor in two children, who developed severe acute pancreatitis with considerable complement activation after allogeneic haematopoietic stem cell transplantation.

Methods-Both children showed clinical features resembling those observed in capillary leakage syndrome. In both patients, treatment with $\mathrm{C} 1$ esterase inhibitor concentrate contributed to a rapid clinical stabilisation.

Conclusions-These observations strongly support the proposed pathophysiological concept that early treatment with $\mathrm{C} 1$ esterase inhibitor interferes with the activation of the complement system in acute pancreatitis. Inhibition of complement activation prevents its adverse effects on vascular function and permeability, and thus stabilises intravascular fluid status and prevents multiorgan failure in acute pancreatitis.

(Gut 1999;45:733-736)

Keywords: acute pancreatitis; capillary leakage syndrome; complement system; kallikrein-bradykinin system; C1 esterase inhibitor.

Acute pancreatitis is characterised by inflammation with activation of digestive proenzymes including proteases, lipase, and amylase, leading to autodigestive destruction of the pancreatic parenchyma and the peripancreatic adipose tissue. Proteases activate numerous enzyme cascades including the complement, kallikrein-bradykinin, and coagulation systems. ${ }^{1-4}$ In severe acute pancreatitis, the ensuing consequences are vascular dilatation, increase in capillary permeability with local oedema, and systemic effects such as third spacing of fluids, finally leading to cardiovascular destabilisation and multiorgan failure despite intensive care and surgical treatment of the pancreatitis. ${ }^{5}$

Therefore various inhibitors of these protease cascades have been studied for their therapeutic effects in acute pancreatitis. ${ }^{26} \mathrm{C} 1$ esterase inhibitor is the most important inhibitor of the classical pathway of complement activation and a major inhibitor of kallikrein. ${ }^{2} 7$ A recent study by Yamaguchi et al reported promising results for a combined treatment with $\mathrm{C} 1$ esterase inhibitor and antithrombin in severe acute experimental pancreatitis. To our knowledge, the effects of C1 esterase inhibitor treatment of acute pancreatitis in humans have not yet been studied. Furthermore patients with acute pancreatitis following stem cell transplantation have not yet been investigated for complement activation.

We report on two children who developed severe acute pancreatitis after allogeneic haematopoietic stem cell transplantation (STCT). Both patients had signs of activation of the complement cascade, and both showed a significant response to early treatment with $\mathrm{C} 1$ esterase inhibitor.

\section{Patient 1}

This 9 year old boy suffered from a relapsed $\mathrm{T}$ lineage acute lymphoblastic leukaemia, for which he had undergone allogeneic peripheral STCT from his sister. The conditioning treatment contained cyclophosphamide, etoposide, and total body irradiation with 12 Gy, with additional irradiation of the testes because of previous testicular relapse. As there was an HLA mismatch at the MHC class I (B61 versus B51), an intensified graft versus host disease (GvHD) prophylaxis was administered with cyclosporin A (at therapeutic serum levels of $300 \mathrm{ng} / \mathrm{ml}$ ), methotrexate (days 1, 3, and 6), and an antibody against the interleukin 2 receptor (Leucotac; Biotest, Dreieich, Germany).

During marrow aplasia in the first three weeks after transplantation, he had developed severe pseudomembranous colitis with paralytic ileus caused by Clostridium difficile infection. After engraftment on day +21 , he had shown mild GvHD of the skin (temporary grade 2 ). In the later phase after transplanta-

Abbreviations used in this paper: GvHD, graft versus host disease; MHC, major histocompatibility complex; STCT, haematopoietic stem cell transplantation. 
tion, he repeatedly suffered from fatigue and severe heartburn-like symptoms. On day +118 , oesophagogastroscopy and colonoscopy were performed. Histopathological examination of the biopsy specimens disclosed severe ulcerous oesophagitis and residual changes after pseudomembranous colitis. There were no histological signs of acute or chronic GvHD. Multiple immunohistochemical, microbiological, and virological examinations did not provide any evidence that an infectious agent caused the oesophagitis.

One day later he developed severe pancreatitis with an increase in serum lipase from normal levels to $7620 \mathrm{U} / 1$ (normal <190 U/1) and in serum amylase from normal levels to $1476 \mathrm{U} / 1$ (normal <120 U/1). His heart rate was 175 beats/min with normal blood pressure. Central venous pressure was decreased $(0 \mathrm{~mm}$ $\mathrm{Hg})$. $\mathrm{He}$ was anuric.

In addition, this patient showed further prognostically unfavourable parameters, including metabolic acidosis $(\mathrm{pH} 7.0$, base excess $-24 \mathrm{mmol} / \mathrm{l}, \mathrm{pCO}_{2} 22 \mathrm{~mm} \mathrm{Hg}$ ), hyperglycaemia (glucose $736 \mathrm{mg} / \mathrm{dl}$ ), C reactive protein $290 \mathrm{mg} / 1$ (normal <5 mg/l), and white blood cell count $32600 / \mu 1$. Coagulation tests were within normal limits, but D-dimer levels were moderately elevated to $3.6 \mathrm{mg} / 1$ (normal $<0.5$ $\mathrm{mg} / \mathrm{l}$ ). Antithrombin activity was $84 \%$ of normal control (normal $>70 \%$ ). Complement studies showed considerable activation of the complement system, indicated by high levels of complement factor $\mathrm{C} 5 \mathrm{a}(1.51 \mu \mathrm{g} / \mathrm{l}$; normal $<0.75 \mu \mathrm{g} / \mathrm{l})$. Ultrasound examination showed pronounced ascites in the upper abdomen and the pelvis, a small left pleural effusion, and a previously unknown concrement, but no sludge in the gall bladder. Computed tomography showed a swelling of the pancreatic head and appreciable ascites, normal structure of the extrahepatic biliary tract, and no concrements near the ampulla of Vater.

After admission to the intensive care unit, sodium bicarbonate, insulin, and intravenous colloidal fluids were administered. A nasogastric tube was installed. Dopamine was infused at a rate of $4 \mu \mathrm{g} / \mathrm{kg} / \mathrm{min}$. Even though multiple blood cultures were negative, he received broad antibiotic treatment with piperacilline/ tazobactam, vancomycin, metronidazole, and fluconazole for initially suspected septicaemia.

As the clinical presentation of this patient closely resembled that of capillary leakage syndrome, he was enrolled on a compassionate treatment protocol according to the treatment protocol for capillary leakage syndrome following STCT, which had previously been recognised by the local ethics committee. He was treated with $\mathrm{C} 1$ esterase inhibitor concentrate (Berinert; Centeon, Liederbach, Germany; cumulative dose $180 \mathrm{U} / \mathrm{kg}$ over four days ${ }^{7}$ ), starting within 12 hours of the onset of clinical symptoms of pancreatitis.

Soon after the first dose of C1 esterase inhibitor, his clinical condition improved significantly. Central venous pressure rose to normal levels, urine production recovered, and ascites and pleural effusions resolved almost completely, as documented by ultrasound examination on the following day, which showed only minor residual ascites in the cavity of Douglas. C5a levels returned to normal within one day.

Three weeks later he developed a pseudocyst in the pancreatic head, which was first noticed by ultrasound and confirmed by computed tomography. The pseudocyst healed over three months on total parenteral nutrition. At the time of this report (25 months after the acute pancreatitis), the boy remains in remission from leukaemia with no recurrence of pancreatitis.

\section{Patient 2}

This 9 year old girl had developed secondary myelodysplastic syndrome three years after radiochemotherapy for acute lymphoblastic leukaemia. She had been transplanted with peripheral stem cells from an HLA-identical unrelated donor (serologically identical in MHC class I, identical in molecular genetic analysis of MHC class II). The conditioning treatment consisted of total body irradiation (12 Gy), cyclophosphamide, and etoposide. GvHD prophylaxis was performed with cyclosporin A, methotrexate, and an antibody against interleukin 2 receptor as described for patient 1. During the early phase after transplantation, she developed laboratory signs of pancreatitis (maximum serum lipase $600 \mathrm{U} / 1$ on day +21 , maximum serum amylase $326 \mathrm{U} / 1$ on day +22 ; normalisation occurred within 10 days), with only mild clinical symptoms such as moderate abdominal pain. Ultrasound examination of the pancreas was normal. At that time there was evidence of generalised cytomegalovirus infection (positive polymerase chain reaction in blood, bone marrow, and bronchoalveolar lavage), and treatment with ganciclovir was started.

On day +71 after transplantation, she was readmitted to hospital with mild diarrhoea (stool volume $<500 \mathrm{ml} /$ day, no occult blood, no causative infectious agent on microbiological and virological examination), nausea, vomiting, and cramping abdominal pain. She showed clinical signs of mild GvHD of the skin (grade I, affecting less than $25 \%$ of body surface area). She was in severely reduced clinical condition with hyperventilation and tachycardia with arterial hypotension (respiratory rate $50 / \mathrm{min}$, heart rate 170 beats/min, minimum blood pressure $64 / 43 \mathrm{~mm} \mathrm{Hg}$ ), oliguria $(1.1 \mathrm{ml} / \mathrm{kg} / \mathrm{h}$; 24 hour fluid balance $+827 \mathrm{ml}$ ). Soon after admission she deteriorated rapidly, developed repeated generalised epileptic seizures, and was transferred to the intensive care unit, where she had to be mechanically ventilated because of respiratory failure caused by the epileptic state and anticonvulsive medication.

Laboratory investigations were compatible with severe acute pancreatitis, showing a rapid increase in serum lipase to $4730 \mathrm{U} / 1$ within 12 hours. Maximum serum amylase was $2270 \mathrm{U} / 1$. In addition, the girl had a severe metabolic acidosis ( $\mathrm{pH}$ 6.99, $\mathrm{BE}-22 \mathrm{mmol} / 1, \mathrm{pCO}_{2} 30 \mathrm{~mm}$ $\mathrm{Hg}$ ) caused by appreciable hyperglycaemia (maximum serum glucose $20 \mathrm{~g} / \mathrm{l}$ ) Coagulation tests were within the normal range; antithrombin activity was $88 \%$ of normal control. 
Analysis of the complement system showed considerable complement activation (C5a 1.82 $\mu \mathrm{g} / \mathrm{l}$; normal $<0.75 \mu \mathrm{g} / \mathrm{l})$. Polymerase chain reaction for cytomegalovirus was negative. Multiple blood cultures remained sterile. Ultrasound and computed tomography showed pronounced swelling and oedema of the pancreas. The ultrasound of the gall bladder was normal.

After admission to the intensive care unit, the metabolic acidosis and hyperglycaemia were treated. Epileptic seizures ceased after infusion of phenytoin. Broad antibiotic treatment with piperacilline/tacobactam and teicoplanin was started. Despite treatment with colloidal fluids, dopamine (3 $\mu \mathrm{g} / \mathrm{kg} / \mathrm{min})$, furosemide, and etacrynic acid, the oliguria persisted. C1 esterase inhibitor treatment was started as described previously for patient 1 . Two hours after the first C1 esterase inhibitor dose, cardiovascular stabilisation was achieved and urine production increased $(3.2 \mathrm{ml} / \mathrm{kg} / \mathrm{h}$, 24 hour fluid balance after start of $\mathrm{C} 1$ esterase inhibitor $-540 \mathrm{ml}$ ).

In the following weeks the patient repeatedly suffered from nausea and abdominal pain. There was only faint GvHD of the skin and no signs of intestinal or hepatic GvHD. Prolonged elevation of serum lipase activity was observed. Computed tomography did not show any evidence of a pancreatic pseudocyst. Nevertheless, the clinical symptoms were attributed to smouldering pancreatitis with undulating increases in amylase and lipase.

Two months after this episode of acute pancreatitis, this patient suffered a relapse of myelodysplastic syndrome and died on day 146 after transplantation.

\section{Discussion}

Acute pancreatitis has been observed in $3.5-4.4 \%$ of paediatric and adult bone marrow transplantation patients. ${ }^{8} 9$ Moreover, $27 \%$ of patients who die after STCT show signs of acute pancreatitis at autopsy, suggesting an additional impact of acute pancreatitis on morbidity and mortality in severely ill transplantation patients. ${ }^{10}$ The increasing incidence of STCT means that consultant gastroenterologists will be confronted with this clinical situation more often.

The pathophysiology of acute pancreatitis after transplantation is multifactorial and includes transplantation related toxicity (conditioning chemotherapy and irradiation), acute GvHD, bacterial and viral infections-for example, cytomegalovirus infections-, cholestasis due to long term parenteral nutrition, and defined medications - for example, cyclosporin A, glucocorticosteroids. Severe acute GvHD (grade 3-4) and hepatic GvHD seem to be the major risk factors for acute pancreatitis after STCT. ${ }^{10}$ Sonographic studies showed a high prevalence of gall bladder sludge and gallstones in transplant patients (11 of 18 patients). ${ }^{11}{ }^{12}$ The sludge showed a unique chemical composition with large amounts of calcium bilirubinate and a protein-lipid complex. ${ }^{12}$ However, the prevalence of gall bladder sludge was comparable with that observed in association with prolonged fasting and total parenteral nutrition, and the authors reported no association with acute cholecystitis or pancreatitis. ${ }^{11}$ Moreover, in the clinicopathological study, all transplant patients, with or without pancreatitis, had gall bladder sludge at autopsy. ${ }^{10}$ Therefore it was concluded that gall bladder sludge may be of only minor relevance in the pathophysiology of acute pancreatitis after STCT. In this study, pancreatic infection played a minor role also ( $10 \%$ of pancreatitis patients). ${ }^{10}$ In summary, in most patients, pancreatitis cannot be attributed to a single pathogenetic factor. ${ }^{8}$ The course of pancreatitis in these patients is often complicated by the severe immunosuppression and other transplantation related complications - for example, chronic renal insufficiency-and therefore the prognosis of pancreatitis after STCT is moderate.

In our patients also, the cause of the acute pancreatitis remains unclear. In patient 1 , a chologeneic cause may be conceivable, as a gall bladder stone was seen on ultrasound, but the computed tomography scan showed evidence of neither dilatation of the extrahepatic bile ducts nor concrements near the papilla of Vater. In patient 2, the first episode of mild pancreatitis was associated with systemic cytomegalovirus infection, but on the occasion reported here, there was no proof of cytomegalovirus re-activation. In both patients, a systemic infection causing pancreatitis seemed unlikely, because both were afebrile, blood cultures were sterile, and serological tests for bacteriological and virological agents and polymerase chain reaction for cytomegalovirus were all normal.

Irrespective of the contributing pathogenetic factors, severe acute pancreatitis is characterised by self perpetuating activation of proteases leading to autodigestive destruction of the pancreas and the surrounding tissue. These proteases activate numerous enzyme cascades such as the complement, the kallikrein-bradykinin, and the coagulation systems. A recent study showed that liberation of trypsin in caerulein induced pancreatitis in rats leads to cleavage of components of the complement system. ${ }^{13}$ Other studies of acute pancreatitis in humans showed activation of both the classical and alternative pathway of the complement system. ${ }^{5}$ Experimental data show that complement activation results in liberation of the anaphylatoxin C5a, which precedes neutrophil lung sequestration, the formation of angioedema, and capillary leakage syndrome. ${ }^{79}$ In this experimental setting, the complement induced neutrophil lung sequestration could be reversed by soluble complement receptor CR 1 . $^{9}$

Other studies have shown the influence of the complement system on oedema formation. In acute pancreatitis induced by ligation of the pancreatic duct in mice lacking the circulating complement factor $\mathrm{C} 5$, oedema formation was significantly reduced. ${ }^{13}$ However, until now the pathophysiological impact of complement activation in acute pancreatitis in humans has not been studied in detail.

Encouraged by these experimental observations, various workers have studied the effect of 
prophylactic and therapeutic administration of C1 esterase inhibitor in experimental models of acute pancreatitis. ${ }^{214-16}$ Indubitable beneficial effects of prophylactic administration were demonstrated, whereas the results of therapeutic administration were ambiguous, indicating the importance of early therapeutic intervention to disrupt the pathophysiological mechanisms at an early stage.

Yamaguchi et $a l^{2}$ have recently reported promising results for the combined treatment of $\mathrm{C} 1$ esterase inhibitor with antithrombin concentrate for severe haemorrhagic pancreatitis in rats, which was induced by retrograde injection of sodium taurocholate into the pancreatic duct. They concluded that activation of the complement system plays a central role in the pathophysiology of the systemic effects of severe acute pancreatitis.

The clinical pictures of acute pancreatitis and capillary leakage syndrome, which has also been observed in sepsis and in the early stages after STCT, share common features: severe intravascular hypovolaemia due to third spacing into serous cavities leads to cardiovascular destabilisation and finally multiorgan failure. In both disorders we and others observed laboratory signs of considerable complement activation. ${ }^{5}$ In both the cases of capillary leakage syndrome studied by us and in the patients described above, C1 esterase inhibitor treatment resulted in rapid cardiovascular stabilisation and resolution of the serous effusions. These observations are in good agreement with the experimental findings reported by Yamaguchi et $a .^{2}$

In the patients described by us we cannot of course clearly distinguish between the respective therapeutic impacts of $\mathrm{C} 1$ esterase inhibitor treatment and the conventional supportive treatment. Nevertheless, the course of the disease and the response to treatment, which showed significant clinical improvement shortly after the initiation of $\mathrm{C} 1$ esterase inhibitor treatment in both patients, leads us to the conclusion that this treatment at least strongly enhances the efficacy of conventional treatment in acute pancreatitis. Similar therapeutic effects have been documented for C1 esterase inhibitor treatment of capillary leakage syndrome after STCT. $^{7}$ We observed no side effects of $\mathrm{C} 1$ esterase inhibitor treatment.

Patients in the early phase after STCT are at increased risk of developing disorders associated with complement activation such as capillary leakage syndrome. ${ }^{7}$ This syndrome is observed at the beginning of leucocyte engraftment, indicating the pathophysiological role of the leucocytes in the mediation of complement activation. However, capillary leakage syndrome with complement activation has also been observed in non-transplant patients. ${ }^{5}{ }^{17}$

We cannot determine to what extent the transplantation contributed to the development of the clinical picture and the complement activation in our patients. However, in both patients the transplantation had been performed several weeks before. As complement activation has been shown in other patients with acute pancreatitis, ${ }^{5}$ this pathophysiologi- cal phenomenon is obviously not confined to the STCT associated pancreatitis and deserves more attention in the clinical evaluation and planning of treatment of patients with severe acute pancreatitis.

In summary, our observations support the hypothesis that complement activation may be of central importance in mediating the often fatal systemic effects in severe acute pancreatitis. In our patients, treatment with $\mathrm{C} 1$ esterase inhibitor did not significantly influence the local inflammation and autodigestive destruction of the pancreas, as both patients developed chronic local complications. However, blockage of the complement system by $\mathrm{C} 1$ esterase inhibitor in combination with conventional treatment reversed cardiovascular instability and acute renal failure resulting from third spacing of fluids. Therefore the relevance of complement activation and its influence on cardiovascular status and diuresis should be studied systematically in acute pancreatitis in humans. The therapeutic benefit of $\mathrm{C} 1$ esterase inhibitor in pancreatitis should be evaluated in future clinical trials.

The bone marrow transplantation programme is supported by the Elterninitiative Kinderkrebsklinik Duesseldorf.

1 Blackberg M, Ohlsson K. Studies on the release of tissue kallikrein in experimental pancreatitis in the pig. Eur Surg Res 1994;26:116-24.

2 Yamaguchi $\mathrm{H}$, Weidenbach $\mathrm{H}$, Luhrs $\mathrm{H}$, et al. Combined treatment with $\mathrm{C} 1$ esterase inhibitor and antithrombin III improves survival in severe acute experimental pancreatitis. Gut 1997;40:531-5.

3 Gross V, Leser HG, Heinisch A, et al. Inflammatory mediators and cytokines-new aspects of the pathophysiology and assessment of severity of acute pancreatitis? Hepatogastroenterology 1993;40:522-30.

4 Lasson A, Ohlsson K. Consumptive coagulopathy, fibrinolysis and protease-antiprotease interactions during acute human pancreatitis. Thromb Res 1986;41:167-83.

5 Lasson A, Laurell AB, Ohlsson K. Correlation among complement activation, protease inhibitors, and clinical course in acute pancreatitis in man. Scand $\mathcal{F}$ Gastroenterol 1985;20: $335-45$

6 Lerch MM, Weidenbach H, Gress TM, et al. Effect of kinin inhibition in experimental acute pancreatitis. Am f Physiol 1995;269:G490-9.

7 Nürnberger W, Heying R, Burdach S, et al. C1 esterase inhibitor concentrate for capillary leakage syndrome following bone marrow transplantation. Ann Hematol 1997;75:95-101.

8 Shore T, Bow E, Greenberg H, et al. Pancreatitis post-bone marrow transplantation. Bone Marrow Transplant 1996;17: $1181-4$

9 Werlin SL, Casper J, Antonson D, et al. Pancreatitis associated with bone marrow transplantation in children. Bone Marrow Transplant 1992;10:65-9.

$10 \mathrm{Ko}$ CW, Gooley T, Schoch HG, et al. Acute pancreatitis in marrow transplant patients: prevalence at autopsy and risk factor analysis. Bone Marrow Transplant 1997;20:1081-6.

11 Jacobson AF, Teefey SA, Lee SP, et al. Frequent occurrence of new hepatobiliary abnormalities after bone marrow transplantation: results of a prospective study using scintigtransplantation: results of a prospective study using scintig-

12 Ko CW, a sonography. Am f Gastroenterol 1993;88:1044-9. sition of gallbladder sludge in patients after marrow transsition of gallbladder sludge in patients after marr
plantation. Am $\mathcal{F}$ Gastroenterol 1996;91:1207-10.

13 Acioli JM, Isobe M, Kawasaki S. Early complement system activation and neutrophil priming in acute pancreatitis: activation and neutrophil priming in acute pan

14 Kald B, Kald A, Ihse I, et al. Release of platelet-activating factor in acute experimental pancreatitis. Pancreas 1993;8: 440-2.

15 Lium B, Ruud TE, Pillgram-Larsen J, et al. Sodium taurocholate-induced acute pancreatitis in pigs. Pathomorphological studies of the pancreas in untreated animals and animals pretreated with high doses of corticosteroids or protease inhibitors. Acta Pathologica Microbiologica Immunologica Scandinavica [A] 1987;95:377-82.

16 Ruud TE, Aasen AO, Pillgram-Larsen J, et al. Effects on peritoneal proteolysis and hemodynamics of prophylactic infusion with C1 inhibitor in experimental acute pancreatitis. Scand $\mathcal{F}$ Gastroenterol 1986;21:1018-24.

17 Hazelzet JA, de Groot R, van Mierlo G, et al. Complement activation in relation to capillary leakage in children with septic shock and purpura. Infect Immun 1998;66:5350-6. 